\title{
On 0-1 matrices whose inverses have entries of the same modulus
}

\author{
Xavier Martínez-Rivera*
}

September 16, 2020

\begin{abstract}
A conjecture of Barrett, Butler and Hall may be stated as follows: If $n \geq 3$ and $A \in\{0,1\}^{n \times n}$ (the family of $n \times n \quad 0-1$ matrices) is a nonsingular symmetric matrix, then the following two statements are equivalent: (a) All of the principal minors of $A$ of order $n-2$ are zero; and (b) $A^{-1}$ is a matrix all of whose entries have the same modulus and all of whose diagonal entries are equal. We show that this conjecture holds if $A$ does not have both a zero and a nonzero principal minor of order $n-4$ (if $n \geq 5$ ). The parity of the principal minors of nonsingular symmetric matrices $A \in\{0,1\}^{n \times n}$ whose principal minors of order $n-2$ are all zero is explored, establishing, in particular, that the determinants of such matrices are all even. The aforementioned conjecture was stated in terms of the enhanced principal rank characteristic sequence (epr-sequence) of the matrix $A$, a sequence defined as follows: $\ell_{1} \ell_{2} \cdots \ell_{n}$, where $\ell_{j}$ is either $\mathrm{A}, \mathrm{S}$, or $\mathrm{N}$, based on whether all, some but not all, or none of its principal minors of order $j$ are nonzero. The eprsequences that start with AN but whose fourth letter is not $\mathrm{S}$ (which are of particular relevance for us) are completely characterized; moreover, it is shown that, if $B$ is an arbitrary symmetric matrix with zero diagonal, then the odd-girth of its graph, as well as whether or not its graph is bipartite or an odd cycle, is deducible from its epr-sequence. For an arbitrary (not necessarily symmetric) nonsingular matrix $A \in\{0,1\}^{n \times n}$ with $n \geq 3$, we establish necessary conditions for $A^{-1}$ to be a matrix all of whose entries have the same modulus; examples of such conditions are the following: each row and column of $A$ has an even number of nonzero entries; each entry of $A^{-1}$ is the reciprocal of an even integer; $\operatorname{det}(A)$ is even; the difference between any two rows of $A$, as well as the difference between any two columns of $A$, has an even number of nonzero entries; if $A$ is symmetric, then $A$ has an even number of nonzero diagonal entries; if $A$ is symmetric and $\vec{a}_{k}$ is the $k$ th column of $A$, then $A-\vec{a}_{k} \vec{a}_{k}^{T}$ has an even number of nonzero diagonal entries.
\end{abstract}

Keywords. Equimodular; constant diagonal; enhanced principal rank characteristic sequence; symmetric matrix; minor.

AMS subject classifications. 15B57, 15A15, 15A03, 05C50.

${ }^{*}$ Department of Mathematics and Statistics, University of Victoria, Victoria, BC, V8W 2Y2, Canada (martinez.rivera.xavier@gmail.com). 


\section{Introduction}

We are concerned with nonsingular $0-1$ matrices whose inverses are matrices all of whose entries have the same modulus (absolute value), with our chief concern being a conjecture of Barrett, Butler and Hall [2]. The conjecture states that, if $n \geq 3$ and $A \in \mathbb{R}^{n \times n}$ is a nonsingular symmetric $0-1$ matrix, then the following two statements are equivalent: (i) All of the principal minors of $A$ of order $n-2$ are zero; and (ii) $A^{-1}$ is a matrix all of whose entries have the same modulus and all of whose diagonal entries are equal. Although our primary motivation is the aforementioned conjecture, which is concerned with symmetric matrices, some of our results apply to non-symmetric matrices (see Section 5).

In the interest of keeping our presentation consistent with that of [2], and because the above formulation of the conjecture lacks simplicity, we shall restate the aforementioned conjecture using the terminology used in [2], after introducing the necessary terminology.

For a given positive integer $n,[n]:=\{1,2, \ldots, n\}$. For $B \in \mathbb{R}^{n \times n}$ and $\mu, \omega \subseteq[n]$, $B[\mu, \omega]$ denotes the submatrix of $B$ lying in rows indexed by $\mu$ and columns indexed by $\omega$, and $B(\mu, \omega)$ denotes the submatrix obtained by deleting the rows indexed by $\mu$ and columns indexed by $\omega ; B[\mu, \mu]:=B[\mu]$ and is called a principal submatrix; and $B(\mu, \mu):=B(\mu)$. We say that an $n \times n$ matrix has order $n$. The determinant of a $k \times k$ principal submatrix is a principal minor, and such a minor has order $k$. The rank of a symmetric matrix is called principal (because of a well-known fact, which is encapsulated in Theorem 2.3).

For a given symmetric matrix $B \in \mathbb{R}^{n \times n}$, the enhanced principal rank characteristic sequence (epr-sequence) of $B$ (which was introduced in [5] and motivated by [3]) is $\operatorname{epr}(B)=\ell_{1} \ell_{2} \cdots \ell_{n}$, where

$$
\ell_{j}= \begin{cases}\mathrm{A} & \text { if all of the principal minors of order } j \text { are nonzero; } \\ \mathrm{S} & \text { if some but not all of the principal minors of order } j \text { are nonzero; } \\ \mathrm{N} & \text { if none of the principal minors of order } j \text { are nonzero (i.e., all are zero); }\end{cases}
$$

for convenience, $[\operatorname{epr}(B)]_{j}:=\ell_{j}$, for all $j \in[n]$. Following the convention adopted in [2], for all $k \in[n]$, we say that " $B$ starts with $\ell_{1} \ell_{2} \cdots \ell_{k}$," and that " $B$ ends with $\ell_{k} \ell_{k+1} \cdots \ell_{n}$." For a given sequence $t_{i_{1}} t_{i_{2}} \cdots t_{i_{k}}$ from $\{\mathrm{A}, \mathrm{N}, \mathrm{S}\}, \overline{t_{i_{1}} t_{i_{2}} \cdots t_{i_{k}}}$ indicates that the sequence may be repeated as many times as desired (or it may be omitted entirely).

The set containing all of the matrices in $\mathbb{R}^{m \times n}$ all of whose entries are either $\alpha_{1}, \alpha_{2}, \ldots, \alpha_{k-1}$ or $\alpha_{k}$, for some $\alpha_{1}, \alpha_{2}, \ldots, \alpha_{k} \in \mathbb{R}$, is denoted by $\left\{\alpha_{1}, \alpha_{2} \ldots, \alpha_{k}\right\}^{m \times n}$. In particular, matrices in $\{0,1\}^{m \times n}$ are referred to as $0-1$ matrices. Following the convention adopted in [2], we call a matrix $B \in \mathbb{R}^{n \times n}$ equimodular if all of its entries are of the same modulus; that is, $B$ is equimodular if, for some $\alpha \in \mathbb{R}, B \in\{-\alpha, \alpha\}^{n \times n}$. If the diagonal entries of a given square matrix are all equal, then we say that the matrix has "constant diagonal."

As no real symmetric matrix has NNA or NSA as a subsequence (see [5, Theorem 2.3] and [5, Corollary 2.7]), the following observation was made in [2]:

Observation 1.1. [2, p. 42] Let $n \geq 3$ be an integer and $A \in\{0,1\}^{n \times n}$ be symmetric and nonsingular. Then $A$ ends with NAA if and only if all of the principal minors of $A$ of order $n-2$ are zero. 
Observation 1.1 permits an alternative formulation of the aforementioned conjecture:

Conjecture 1.2. [2, p. 43] Let $n \geq 3$ be an integer and $A \in\{0,1\}^{n \times n}$ be symmetric and nonsingular. Then the following statements are equivalent:

(a) A ends with NAA.

(b) $A^{-1}$ is equimodular with constant diagonal.

For the nonsingular symmetric 0-1 matrix $A$ below, which is juxtaposed with its inverse, $A^{-1}$, the following is true: $A$ ends with NAA $\left(\operatorname{epr}(A)=\right.$ NSNAA) and $A^{-1}$ is equimodular with constant diagonal $\left(A^{-1} \in\left\{-\frac{1}{2}, \frac{1}{2}\right\}^{5 \times 5}\right.$ and each diagonal entry of $A^{-1}$ is equal to $\left.\frac{1}{2}\right)$ :

$$
A=\left(\begin{array}{lllll}
0 & 1 & 0 & 0 & 1 \\
1 & 0 & 1 & 0 & 0 \\
0 & 1 & 0 & 1 & 0 \\
0 & 0 & 1 & 0 & 1 \\
1 & 0 & 0 & 1 & 0
\end{array}\right) ; \quad A^{-1}=\frac{1}{2}\left(\begin{array}{rrrrr}
1 & 1 & -1 & -1 & 1 \\
1 & 1 & 1 & -1 & -1 \\
-1 & 1 & 1 & 1 & -1 \\
-1 & -1 & 1 & 1 & 1 \\
1 & -1 & -1 & 1 & 1
\end{array}\right)
$$

By means of an exhaustive computer search, it was determined by the authors of [2], and announced in [2, p. 53-54], that Conjecture 1.2 holds for $n \leq 9$. Although the conjecture remains unresolved, one of its two directions has already been established:

Remark 1.3. [2, p. 43] In Conjecture 1.2, (b) implies (a).

Remark 1.3 implies that the family of nonsingular symmetric 0-1 matrices whose inverses are equimodular with constant diagonal is a subset of the family of nonsingular symmetric 0-1 matrices that end with NAA. Conjecture 1.2 speculates that these two families are one and the same. Furthermore, Remark 1.3 implies that, to establish Conjecture 1.2 (if it is true), it suffices to show that (a) implies (b); i.e., it suffices to show that the inverse of an arbitrary symmetric $0-1$ matrix that ends with NAA is equimodular with constant diagonal; to show this, it suffices to show that all of the principal minors of order $n-1$ of an arbitrary symmetric matrix in $\{0,1\}^{n \times n}$ that ends with NAA are equal:

Remark 1.4. [2, p. 45] Let $A \in\{0,1\}^{n \times n}$ be a symmetric matrix that ends with NAA. If all of the principal minors of $A$ of order $n-1$ are equal, then $A^{-1}$ is equimodular with constant diagonal.

One of our chief objectives is to establish that, if the matrix $A$ in Conjecture 1.2 satisfies one particular condition, then the conjecture holds; we provide more details regarding this condition at the end of this section, immediately after additional terminology is introduced. We shall also cast more light upon the two families of nonsingular symmetric 0-1 matrices that have been conjectured (in Conjecture 1.2) to be one and the same: The family whose members end with NAA and the family whose members are nonsingular matrices whose inverses are equimodular with constant diagonal.

For us, all graphs are simple, undirected and loopless. The cycle graph on $n \geq 3$ vertices is denoted by $C_{n}$, and $C_{n}$ is odd (respectively, even), if $n$ is odd (respectively, 
even). For a given graph $G$, its number of vertices is its order; the odd-girth of $G$ is defined to be the length (number of vertices, or edges) of a shortest odd cycle in $G$, and, if no such cycle exists, then it is defined to be $\infty$; and the $0-1$ adjacency matrix of $G$ is denoted by $A(G)$. A given square matrix all of whose diagonal entries are zero is said to have "zero diagonal." With a given symmetric matrix $B=\left[b_{i j}\right] \in \mathbb{R}^{n \times n}$ that has zero diagonal, we associate a graph, which we denote by $G(B)$, whose vertex set is $[n]$, and whose edges are determined as follows: The graph $G(B)$ has an edge between the vertices $i, j \in[n]$ if and only if $b_{i j}$ is nonzero.

For a given matrix $B \in \mathbb{R}^{n \times n}$ with a nonsingular principal submatrix $B[\mu]$, recall that the Schur complement of $B[\mu]$ in $B$ is the matrix $B / B[\mu]:=B\left[\mu^{c}\right]-B\left[\mu^{c}, \mu\right](B[\mu])^{-1} B\left[\mu, \mu^{c}\right]$, where $\mu^{c}=[n] \backslash \mu$ (see, for example, [9]). The Kronecker product (also known as tensor product) of two given matrices $B$ and $C$ is denoted by $B \otimes C$. The matrices $B$ and $C$ are said to be permutationally similar if there exists a permutation matrix $P$ such that $C=P^{T} B P$. By $I_{n}, O_{m, n}$ and $J_{n}$ we denote, respectively, the identity matrix of order $n$, the $m \times n$ zero matrix and the $n \times n$ matrix all of whose entries are equal to 1 ; furthermore, $O_{n, n}:=O_{n}$.

All of the matrices treated in this paper are real (i.e., they are over $\mathbb{R}$ ). However, we shall, at one point in Section 4, analyze a given (real) 0-1 matrix by treating it as a matrix over the field of order 2 , which we shall denote by $\mathbb{Z}_{2}$.

The purpose of Section 2 is merely to list some known results that will be cited in the subsequent pages. In Section 3, our focus is on epr-sequences that start with AN (which are of particular relevance to Conjecture 1.2), as well as on epr-sequences that start with N: The epr-sequences that start with AN but whose fourth term is not $\mathrm{S}$ are completely characterized; and it is shown that, if $B$ is an arbitrary symmetric matrix with zero diagonal (i.e., a matrix that starts with N), then the odd-girth of $G(B)$, as well as whether or not $G(B)$ is bipartite or an odd cycle, is deducible from $\operatorname{epr}(B)$. Section 4 is devoted to symmetric $0-1$ matrices that end with NAA (i.e., to the matrices in the first of the two families associated with Conjecture 1.2); in particular, it is established that Conjecture 1.2 holds if $A$ does not have both a zero and a nonzero principal minor of order $n-4$ (if $n \geq 5$ ); moreover, motivated by Remark 1.4, attention is paid to the parity of the principal minors of these matrices, especially to the parity of their principal minors of order $n-1$ and the parity of their determinants, with the latter of which shown to all be even. Section 5 focuses on (not necessarily symmetric) nonsingular 0-1 matrices whose inverses are equimodular, due to their relation with the second of the two families associated with Conjecture 1.2 (namely, the family of nonsingular symmetric 0-1 matrices whose inverses are equimodular with constant diagonal); for an arbitrary (not necessarily symmetric) nonsingular matrix $A \in\{0,1\}^{n \times n}$ with $n \geq 3$, we establish necessary conditions for $A^{-1}$ to be equimodular; examples of such conditions are the following: each row and column of $A$ has an even number of nonzero entries; each entry of $A^{-1}$ is the reciprocal of an even integer; $\operatorname{det}(A)$ is even; the difference between any two rows of $A$, as well as the difference between any two columns of $A$, has an even number of nonzero entries; if $A$ is symmetric, then $A$ has an even number of nonzero diagonal entries; if $A$ is symmetric and $\vec{a}_{k}$ is the $k$ th column of $A$, then $A-\vec{a}_{k} \vec{a}_{k}^{T}$ has an even number of nonzero diagonal entries. 


\section{Some known results}

The purpose of this section is to list some known results (of which most are about epr-sequences) that will be cited in the remaining pages; some of them are assigned names by which they will be referenced. Results that are restricted to the field $\mathbb{R}$ may hold over other fields (most results are stated in the context of $\mathbb{R}$ merely for simplicity). The first result listed is well-known (and elementary):

Theorem 2.1. Let $B \in \mathbb{R}^{n \times n}$ be nonsingular and $B^{-1}=\left[\beta_{i j}\right]$. Then, for all $i, j \in[n]$,

$$
\beta_{i j}=(-1)^{i+j} \frac{\operatorname{det}(B(\{j\},\{i\}))}{\operatorname{det}(B)} \quad \text { and } \quad \beta_{i i}=\frac{\operatorname{det}(B(\{i\}))}{\operatorname{det}(B)} .
$$

The next result, which is also well-known, is concerned with Schur complements.

Theorem 2.2. (Schur Complement Theorem.) Let $B \in \mathbb{R}^{n \times n}, \operatorname{rank}(B)=r, \mu \subset[n]$, $\mu^{c}=[n] \backslash \mu$ and $|\mu|=k$. Suppose that $B[\mu]$ is nonsingular, and let $C=B / B[\mu]$. Then the following statements hold:

(i) $C$ is an $(n-k) \times(n-k)$ matrix.

(ii) If $B$ is symmetric, then $C$ is symmetric.

(iii) [9, p. 14] $\operatorname{rank}(C)=r-k$.

(iv) [9, Theorem 1.2] If $B$ is nonsingular, then $C$ is nonsingular and $C^{-1}=B^{-1}\left[\mu^{c}\right]$.

(v) [4, p. 771] If the indexing of $C$ is inherited from $B$, then, for all $\gamma \subseteq \mu^{c}$,

$$
\operatorname{det}(C[\gamma])=\frac{\operatorname{det}(B[\gamma \cup \mu])}{\operatorname{det}(B[\mu])} .
$$

The following is also a well-known fact (see, for example, [1, Theorem 1.1]), and it states that the rank of an arbitrary symmetric matrix $B$ is equal to the order of a largest nonsingular principal submatrix of $B$, which is why, in Section 1, we adopted the convention of calling the rank of a symmetric matrix "principal."

Theorem 2.3. Let $B \in \mathbb{R}^{n \times n}$ be symmetric. Then $\operatorname{rank}(B)=\max \{|\mu|: \operatorname{det}(B[\mu]) \neq 0\}$, where the maximum over the empty set is defined to be 0 .

The next result follows from the Schur Complement Theorem.

Theorem 2.4. [6, Corollary 1.11] Let $B \in \mathbb{R}^{n \times n}$ be symmetric, $\operatorname{epr}(B)=\ell_{1} \ell_{2} \cdots \ell_{n}$, $\mu \subset[n]$ and $|\mu|=k$. Suppose that $B[\mu]$ is nonsingular, and let $C=B / B[\mu]$ and $\operatorname{epr}(C)=\ell_{1}^{\prime} \ell_{2}^{\prime} \cdots \ell_{n-k}^{\prime}$. If $\ell_{j+k} \in\{\mathrm{A}, \mathrm{N}\}$, then $\ell_{j}^{\prime}=\ell_{j+k}$, for all $j \in[n-k]$.

The epr-sequence of the inverse of an arbitrary nonsingular symmetric matrix is deducible from that of the given matrix: 
Theorem 2.5. [5, Theorem 2.4] (Inverse Theorem.) Let $B \in \mathbb{R}^{n \times n}$ be symmetric. Suppose that $B$ is nonsingular. If $\operatorname{epr}(B)=\ell_{1} \ell_{2} \cdots \ell_{n-1} \mathrm{~A}$, then $\operatorname{epr}\left(B^{-1}\right)=\ell_{n-1} \ell_{n-2} \cdots \ell_{1} \mathrm{~A}$.

The next result allows one to deduce some of the terms in the epr-sequence of a principal submatrix of an arbitrary symmetric matrix, provided that the epr-sequence of the latter is known.

Theorem 2.6. [5, Theorem 2.6] (Inheritance Theorem.) Let $B \in \mathbb{R}^{n \times n}$ be symmetric, $m \leq n$ and $1 \leq i \leq m$. Then the following statements hold:

1. If $[\operatorname{epr}(B)]_{i}=\mathrm{N}$, then $[\operatorname{epr}(C)]_{i}=\mathrm{N}$, for all $m \times m$ principal submatrices $C$.

2. If $[\operatorname{epr}(B)]_{i}=\mathrm{A}$, then $[\operatorname{epr}(C)]_{i}=\mathrm{A}$, for all $m \times m$ principal submatrices $C$.

3. If $[\operatorname{epr}(B)]_{m}=\mathrm{S}$, then there exist $m \times m$ principal submatrices $C_{A}$ and $C_{N}$ of $B$ such that $\left[\operatorname{epr}\left(C_{A}\right)\right]_{m}=\mathrm{A}$ and $\left[\operatorname{epr}\left(C_{N}\right)\right]_{m}=\mathrm{N}$.

4. If $i<m$ and $[\operatorname{epr}(B)]_{i}=\mathrm{S}$, then there exists an $m \times m$ principal submatrix $C_{S}$ such that $\left[\operatorname{epr}\left(C_{S}\right)\right]_{i}=\mathrm{S}$.

If the epr-sequence of an arbitrary $n \times n$ symmetric matrix $B$ is known, then there is a way to append a row and a column to $B$ so that the epr-sequence of the resulting $(n+1) \times(n+1)$ matrix "inherits" the first letter of $\operatorname{epr}(B)$ as well as any Ns (and Ss) in $\operatorname{epr}(B)$ :

Observation 2.7. [5, Observation 2.19] Let $B=\left[b_{i j}\right] \in \mathbb{R}^{n \times n}$ be symmetric and $\operatorname{epr}(B)=$ $\ell_{1} \ell_{2} \cdots \ell_{n}$. Let $\vec{x}$ be the nth column of $B$,

$$
B^{\prime}=\left(\begin{array}{c|c}
B & \vec{x} \\
\hline \vec{x}^{T} & b_{n n}
\end{array}\right)
$$

and $\operatorname{epr}\left(B^{\prime}\right)=\ell_{1}^{\prime} \ell_{2}^{\prime} \cdots \ell_{n+1}^{\prime}$. Then $\operatorname{epr}\left(B^{\prime}\right)=\ell_{1} \ell_{2}^{\prime} \cdots \ell_{n}^{\prime} \mathrm{N}$. Moreover, for $2 \leq j \leq n$, the following statements hold: If $\ell_{j}=\mathrm{N}$, then $\ell_{j}^{\prime}=\mathrm{N}$; and, if $\ell_{j} \in\{\mathrm{A}, \mathrm{S}\}$, then $\ell_{j}^{\prime}=\mathrm{S}$.

If there are two consecutive Ns in the epr-sequence of a symmetric matrix, then each letter in the sequence from that point forward is $\mathrm{N}$ :

Theorem 2.8. [5, Theorem 2.3] (NN Theorem.) Let $B \in \mathbb{R}^{n \times n}$ be symmetric and $\operatorname{epr}(B)=\ell_{1} \ell_{2} \cdots \ell_{n}$. Suppose that $\ell_{k}=\ell_{k+1}=\mathrm{N}$, for some $k$. Then, for all $j \geq k$, $\ell_{j}=\mathrm{N}$.

Theorem 2.9. [5, Corollary 2.7 \& Theorem 2.14] Let $B \in \mathbb{R}^{n \times n}$ be symmetric. Then none of the following are subsequences of $\operatorname{epr}(B)$ :

NSA; NAN; NAS.

We shall reference Theorem 2.9 by saying, for example, that "NSA is forbidden" (likewise with NAN and NAS).

We conclude this section by listing some other known results about epr-sequences. 
Theorem 2.10. [5, Corollary 2.7] Let $B \in \mathbb{R}^{n \times n}$ be symmetric and $\operatorname{epr}(B)=\ell_{1} \ell_{2} \cdots \ell_{n}$. Suppose that, for some $k \in[n-2], \ell_{k} \ell_{k+1} \ell_{k+2}=\mathrm{ASN}$. If $j \geq k+3$, then $\ell_{j} \neq \mathrm{A}$.

Theorem 2.11. [7, Theorem 2.6] Let $B \in \mathbb{R}^{n \times n}$ be symmetric and $\operatorname{epr}(B)=\ell_{1} \ell_{2} \cdots \ell_{n}$. Suppose that $\ell_{1} \ell_{2} \cdots \ell_{n-1}$ contains ANA as a subsequence. Then $\operatorname{epr}(B)$ is of the form $\bar{A} A N A A \bar{A}$.

Theorem 2.12. [7, Theorem 2.7] Let $B \in \mathbb{R}^{n \times n}$ be symmetric and $\operatorname{epr}(B)=\ell_{1} \ell_{2} \cdots \ell_{n}$. Then SNA is not a subsequence of $\ell_{1} \ell_{2} \cdots \ell_{n-2}$.

Theorem 2.13. [7, Proposition 3.5] Let $B \in \mathbb{R}^{n \times n}$ be symmetric with $\operatorname{epr}(B)=\operatorname{ANSNSN} \ell_{7} \cdots \ell_{n}$. Then, for $k \geq 1, \ell_{2 k}=\mathrm{N}$. Furthermore, $\operatorname{epr}(B)$ is of one of the following forms: ANSNSN $\overline{S N} \overline{\mathrm{N}}$ or ANSNSN $\overline{\mathrm{SN}} \mathrm{A}$.

Observation 2.14. [5, Observation 3.1] If $n \geq 3$ is odd, then $\operatorname{epr}\left(A\left(C_{n}\right)\right)=\overline{\mathrm{NSN}}$,

Observe that the matrix $A$ in (1) is $A\left(C_{5}\right)$.

\section{Epr-sequences that start with AN or N}

In this section, results of particular relevance to our results concerning Conjecture 1.2 are established. We start by focusing on epr-sequences that start with AN; the relevance of these is made evident by the following observation (the first statement of which follows from the Inverse Theorem, while second statement follows readily).

Observation 3.1. Let $n \geq 2$ be an integer and $B, C \in \mathbb{R}^{n \times n}$ be symmetric. If $B$ ends with NAA, then $B^{-1}$ starts with AN. If $C \neq O_{n}$ and is equimodular with constant diagonal, then $C$ starts with AN.

We need the next lemma, which generalizes [5, Proposition 2.13(4)].

Lemma 3.2. Let $B \in \mathbb{R}^{n \times n}$ be symmetric, $\operatorname{rank}(B)=r, \mu \subseteq[n]$ and $|\mu|=k$. Suppose that $B[\mu]$ is nonsingular. If $m$ is an integer with $k<m \leq r$, then $B[\mu]$ is contained in either an $m \times m$ or an $(m+1) \times(m+1)$ nonsingular principal submatrix of $B$.

Proof. Suppose that $m$ is an integer with $k<m \leq r$. Let $C=B / B[\mu]$, and assume that the indexing of $C$ is inherited from $B$. By the Schur Complement Theorem, $C \in$ $\mathbb{R}^{(n-k) \times(n-k)}, C$ is symmetric and $\operatorname{rank}(C)=r-k$. Let $\operatorname{epr}(C)=\ell_{1} \ell_{2} \cdots \ell_{n-k}$. Then, as the rank of $C$ is principal, the NN Theorem implies that $\ell_{m-k} \neq \mathrm{N}$ or $\ell_{m+1-k} \neq \mathrm{N}$ (otherwise, $\operatorname{rank}(C)<m-k \leq r-k$, which is a contradiction). Thus, for some $\gamma \subseteq[n] \backslash \mu$ with $|\gamma|=m-k$ or $|\gamma|=m+1-k$, $\operatorname{det}(C[\gamma]) \neq 0$. By the Schur Complement Theorem,

$$
\operatorname{det}(C[\gamma])=\frac{\operatorname{det}(B[\gamma \cup \mu])}{\operatorname{det}(B[\mu])}
$$

implying that $\operatorname{det}(B[\gamma \cup \mu]) \neq 0$. Then, as $|\gamma \cup \mu| \in\{m, m+1\}$, the desired conclusion follows. 
A complete characterization of the epr-sequences that start with AN but whose fourth letter is not $\mathbf{S}$ may now be established.

Theorem 3.3. Let $\sigma=\mathrm{AN}_{3} \ell_{4} \cdots \ell_{n}$ be a sequence from $\{\mathrm{A}, \mathrm{N}, \mathrm{S}\}$ with $\ell_{4} \neq \mathrm{S}$. Then there exists a symmetric matrix $B \in \mathbb{R}^{n \times n}$ with $\operatorname{epr}(B)=\sigma$ if and only if $\sigma$ is of one of the following forms:

\section{AN $\bar{N}, \quad$ ANA $\bar{A}, \quad$ ANSN $\overline{S N} \bar{N}$, ANSN $\overline{S N} A$, ANSNAA, or ANSNSSN $\bar{N}$.}

Proof. Suppose that there exists a symmetric matrix $B \in \mathbb{R}^{n \times n}$ with $\operatorname{epr}(B)=\sigma$. As it is straightforward to verify that the desired conclusion holds for $n \leq 3$ (by referring to [5. Table 1], for example), we assume that $n \geq 4$. If $\ell_{3}=\mathrm{N}$, then the NN Theorem implies that $\sigma=$ ANNN $\bar{N}$. If $\ell_{3}=\mathrm{A}$, then Theorem 2.11 implies that $\sigma=$ ANAA $\bar{A}$. Assume that $\ell_{3}=\mathrm{S}$. By the hypothesis, $\ell_{4} \in\{\mathrm{A}, \mathrm{N}\}$. Then, as NSA is forbidden, $\ell_{4}=\mathrm{N}$, implying that $\sigma$ starts with ANSN. Since it is straightforward to verify that the desired conclusion holds for $n \leq 7$ (by referring to [5, Table 1], for example), we assume that $n \geq 8$. By Theorem 2.12, $\ell_{5} \neq \mathrm{A}$. We consider two cases, based on $\ell_{6}$.

Case 1: $\ell_{6}=\mathrm{N}$.

If $\ell_{5}=\mathrm{N}$, then the NN Theorem implies that $\operatorname{epr}(B)$ is of the form ANSN $\overline{\mathrm{SN}} \overline{\mathrm{N}}$ (with $\overline{\mathrm{SN}}$ vacuous). If $\ell_{5}=\mathrm{S}$, then it follows from Theorem 2.13 that $\operatorname{epr}(B)$ is of one of the following two forms: ANSNSN $\overline{S N} \overline{\mathrm{N}}$ or ANSNSN $\overline{\mathrm{SN}}$ A.

Case 2: $\ell_{6} \neq \mathrm{N}$.

It follows from the NN Theorem that $\ell_{5} \neq \mathrm{N}$. Then, as $\ell_{5} \neq \mathrm{A}, \ell_{5}=\mathrm{S}$. Thus, $\ell_{5} \ell_{6} \in$ $\{\mathrm{SA}, \mathrm{SS}\}$. As NSA is forbidden, $\ell_{5} \ell_{6}=\mathrm{SS}$. Thus, $\operatorname{epr}(B)=\operatorname{ANSNSS}_{7} \cdots \ell_{n}$. Hence, $\operatorname{rank}(B) \geq 6$. We now show that $\operatorname{rank}(B)=6$. Suppose on the contrary that $\operatorname{rank}(B) \geq$ 7. Let $B[\mu]$ be a $6 \times 6$ nonsingular principal submatrix of $B$. By Lemma $3.2, B[\mu]$ is contained in either a $7 \times 7$ or an $8 \times 8$ nonsingular principal submatrix of $B$. Thus, there exists $\omega \subseteq[n] \backslash \mu$ with $1 \leq|\omega| \leq 2$ such that $B[\mu \cup \omega]$ is nonsingular. Let $\operatorname{epr}(B[\mu \cup \omega])=\ell_{1}^{\prime} \ell_{2}^{\prime} \cdots \ell_{6+|\omega|}^{\prime}$. By the Inheritance Theorem, $\ell_{1}^{\prime} \ell_{2}^{\prime} \ell_{3}^{\prime} \ell_{4}^{\prime}=\operatorname{AN} \ell_{3}^{\prime} N$. The nonsingularity of $B[\mu \cup \omega]$ implies that $\ell_{6+|\omega|}^{\prime}=A$. Thus, the NN Theorem implies that $\ell_{3}^{\prime} \neq \mathrm{N}$. Then, as NAN is forbidden, $\ell_{3}^{\prime}=\mathrm{S}$. Thus, either $\operatorname{epr}(B[\mu \cup \omega])=\mathrm{ANSN} \ell_{5}^{\prime} \ell_{6}^{\prime} \mathrm{A}$ or $\operatorname{epr}(B[\mu \cup \omega])=\operatorname{ANSN} \ell_{5}^{\prime} \ell_{6}^{\prime} \ell_{7}^{\prime} \mathrm{A}$. By [5, Table 1], epr $(B[\mu \cup \omega]) \neq \operatorname{ANSN} \ell_{5}^{\prime} \ell_{6}^{\prime} \ell_{7}^{\prime} \mathrm{A}$. Hence, $\operatorname{epr}(B[\mu \cup \omega])=\operatorname{ANSN} \ell_{5}^{\prime} \ell_{6}^{\prime} \mathrm{A}$. It follows from [5, Table 1] that $\operatorname{epr}(B[\mu \cup \omega])=$ ANSNSNA. Thus, each $6 \times 6$ principal submatrix of $B[\mu \cup \omega]$ is singular. Then, as the $6 \times 6$ matrix $B[\mu]$ is a principal submatrix of $B[\mu \cup \omega], B[\mu]$ is singular, which is a contradiction. We conclude that $\operatorname{rank}(B)=6$. Then, as the rank of $B$ is principal, $\ell_{j}=\mathrm{N}$ for all $j \geq 7$, implying that $\operatorname{epr}(B)$ is of the form ANSNSSNN $\bar{N}$.

We now establish the other direction. If $\sigma=\operatorname{AN} \bar{N}$, then $\operatorname{epr}\left(J_{n}\right)=\sigma$ (that follows readily). If $\sigma=\operatorname{ANA} \bar{A}$, then $\operatorname{epr}\left(J_{n}-2 I_{n}\right)=\sigma$ (see [7, Proposition 2.5]). If $\sigma=$ ANSNAA, then [5, Table 1] implies that there exists a symmetric matrix $C^{(0)} \in \mathbb{R}^{6 \times 6}$ with $\operatorname{epr}\left(C^{(0)}\right)=\sigma$. Assume that $\sigma$ is of the form AnSNSSNN $\bar{N}$. By applying Observation 2.7 to $C^{(0)}$, we conclude that there exists a symmetric matrix $C^{(1)} \in \mathbb{R}^{7 \times 7}$ with $\operatorname{epr}\left(C^{(1)}\right)=$ ANSNSSN. Similarly, by applying Observation 2.7 to $C^{(1)}$, we conclude that there exists a symmetric matrix $C^{(2)} \in \mathbb{R}^{8 \times 8}$ with epr $\left(C^{(2)}\right)=$ ANSNSSNN. By continuing in this manner, one concludes that there exists a symmetric matrix $C^{(n-6)} \in \mathbb{R}^{n \times n}$ with $\operatorname{epr}\left(C^{(n-6)}\right)=\operatorname{ANSNSSN\overline {N}}=\sigma$ 
(with $\overline{\mathrm{N}}$ having $n-6$ copies of $\mathrm{N}$ ). Assume that $\sigma$ is of the form ANSN $\overline{\mathrm{SN}} \mathrm{A}$. By Observation 2.14 and the Inverse Theorem, $\operatorname{epr}\left(\left(A\left(C_{n}\right)\right)^{-1}\right)=\sigma$. Finally, assume that $\sigma$ is of the form ANSN $\overline{S N} \overline{\mathrm{N}}$. Because of Observation 2.7, it suffices to establish the desired conclusion in the case where $\overline{\mathrm{N}}$ is assumed to be vacuous. If $\overline{\mathrm{SN}}$ is vacuous (meaning that $\sigma=\mathrm{ANSN}$ ), then, by [5, Table 1], there exists a symmetric matrix $M \in \mathbb{R}^{n \times n}$ with $\operatorname{epr}(M)=\sigma$. Assume that $\overline{\mathrm{SN}}$ is not vacuous (meaning that $\sigma=\operatorname{ANSNSN} \overline{\mathrm{SN}})$. Let $N \in \mathbb{R}^{(n-1) \times(n-1)}$ be a symmetric matrix with $\operatorname{epr}(N)=\operatorname{ANSN} \overline{\operatorname{SN}}$ A (we already showed that $N$ exists). Then, by Observation 2.7, there exists a symmetric matrix $N^{\prime} \in \mathbb{R}^{n \times n}$ with $\operatorname{epr}\left(N^{\prime}\right)=\sigma$.

For the remainder of this section, our attention is turned to epr-sequences that start with N. A natural question is the following one: If $B$ is an arbitrary symmetric matrix with zero diagonal (i.e., a matrix that starts with $\mathrm{N}$ ), what properties of $G(B)$ are deducible from $\operatorname{epr}(B)$ ? Its odd-girth is one such property:

Theorem 3.4. Let $B \in \mathbb{R}^{n \times n}$ be symmetric and $\operatorname{epr}(B)=\ell_{1} \ell_{2} \cdots \ell_{n}$. Suppose that $\ell_{1}=\mathrm{N}$ and let $g$ be the odd-girth of $G(B)$. Then, for all odd $j$ in the interval $[1, n] \cap[1, g), \ell_{j}=\mathrm{N}$. Moreover, $g=\min \left\{j \in[n]: j\right.$ is odd and $\left.\ell_{j} \neq \mathrm{N}\right\}$, where the minimum over the empty set is defined to be $\infty$. In particular, if $G(B)$ is neither bipartite nor an isolated vertex, then $g$ is equal to the order of a smallest nonsingular principal submatrix of $B$ whose order is odd.

Proof. If $n=1$, then $G(B)$ is an isolated vertex, and, therefore, $g=\infty$ and all of the desired conclusions follow readily. Now assume that $n \geq 2$. Let $t=\min \{j \in[n]$ : $j$ is odd and $\left.\ell_{j} \neq \mathrm{N}\right\}$. We divide the proof into two cases:

Case 1: $G(B)$ is bipartite.

It follows, then, that $g=\infty$. Thus, $[1, n] \cap[1, g)=[1, n]$. By the hypothesis, $\ell_{1}=\mathrm{N}$. Since each principal submatrix of $B$ whose order is at least two is bipartite, each principal submatrix of $B$ whose order is odd is singular, implying that $\ell_{j}=\mathrm{N}$, for all odd $j \in[1, n]$, as desired. Hence, $t=\infty=g$, as desired.

Case 2: $G(B)$ is not bipartite.

Since $G(B)$ is not an isolated vertex, it has an odd cycle, implying that $g \geq 3$. Thus, $[1, n] \cap[1, g)=[1, g-1]$. Let $j \in[1, g-1]$ be odd. If $j=1$, then, by the hypothesis, $\ell_{j}=\mathrm{N}$. Assume that $j \geq 3$. Then, as $j<g$, the graph of each $j \times j$ principal submatrix of $B$ does not have any odd cycles, implying that such graphs are all bipartite and of odd order. Thus, $\ell_{j}=\mathrm{N}$. It follows that, for all odd $j$ in the interval $[1, g-1], \ell_{j}=\mathrm{N}$, as desired.

We now show (still within Case 2) that $t=g$. It follows from the final conclusion of the previous paragraph that $t \geq g$. Suppose on the contrary that $t>g$. Then, as $g$ is odd, $\ell_{g}=\mathrm{N}$. Let $B[\mu]$ be a $g \times g$ (principal) submatrix whose graph, $G(B[\mu]$ ), has a cycle of length $g$ (such submatrix exists because $g$ is the odd-girth of $G(B)$ ). Since every cycle of $G(B[\mu])$ is a cycle of $G(B)$, and because $g$ is the odd-girth of $G(B), G(B[\mu])$ does not have any odd cycles whose length is less than $g$. Then, as $g$ is odd and $G(B[\mu])$ is of order $g, G(B[\mu])$ does not have any other edges besides those that are part of its cycle of length $g$ (otherwise, $G\left(B[\mu]\right.$ ) would have a shorter odd cycle). Hence, $G(B[\mu])=C_{g}$. 
It follows (from well-known facts) that $B[\mu]$ is nonsingular, implying that $\ell_{g} \neq \mathrm{N}$, which is a contradiction. We conclude that $t=g$, as desired.

Since a graph whose odd-girth is $\infty$ is either bipartite or an isolated vertex, the last statement of the theorem is readily deducible from the one preceding it.

As a consequence of Theorem 3.4, the following statement holds: If $G$ is a nonbipartite graph of order $n \geq 2$ with odd-girth $g$, then $g$ is equal to the order of a smallest nonsingular principal submatrix of $A(G)$ whose order is odd.

Lemma 3.5. Let $B \in \mathbb{R}^{n \times n}$ be symmetric, $\operatorname{epr}(B)=\ell_{1} \ell_{2} \cdots \ell_{n}$ and $k$ be an odd integer with $1 \leq k \leq n$. Suppose that, for all odd integers $j$ with $1 \leq j \leq k, \ell_{j}=\mathrm{N}$. If $1 \leq k<\operatorname{rank}(B)$, then $\ell_{1} \ell_{2} \cdots \ell_{k}$ is of the form $\mathrm{N} \overline{\mathrm{SN}}$.

Proof. Suppose that $1 \leq k<\operatorname{rank}(B)$. If $k=1$, then the desired conclusion follows by the hypothesis. Assume that $k \geq 3$. It suffices to show that, for all even integers $i$ with $2 \leq i \leq k-1, \ell_{i}=\mathrm{S}$. Let $i$ be an even integer with $2 \leq i \leq k-1$. As $\operatorname{rank}(B)>k$, and because the rank of $B$ is principal, the NN Theorem implies that $\ell_{i} \neq \mathrm{N}$ (otherwise, $\operatorname{rank}(B)<i<k)$. Then, as NAN is prohibited, $\ell_{i}=\mathrm{S}$.

The following fact generalizes some of the results in [5, Observation 3.1], and it implies that the following statement holds: If $B$ is an arbitrary symmetric matrix with zero diagonal (i.e., a matrix that starts with $\mathrm{N}$ ), then whether or not $G(B)$ is bipartite or an odd cycle is deducible from $\operatorname{epr}(B)$.

Theorem 3.6. Let $B \in \mathbb{R}^{n \times n}$ be symmetric and $\operatorname{epr}(B)=\ell_{1} \ell_{2} \cdots \ell_{n}$. Suppose that $\ell_{1}=\mathrm{N}$. Then the following statements hold:

1. $G(B)$ is bipartite if and only if $\operatorname{epr}(B)$ is of one of the following forms: NN $\bar{N}$ or $\overline{\mathrm{NSN}} \mathrm{NA}$

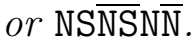

2. $G(B)=C_{2 k+1}$ for some integer $k \geq 1$ if and only if $\operatorname{epr}(B)=\overline{\text { NSNAA. }}$

Proof. We start by establishing statement (1). Suppose that $G(B)$ is bipartite. Thus, $n \geq 2$. If $B=O_{n}$, then, clearly, $\operatorname{epr}(B)$ is of the form $\mathrm{NN} \overline{\mathrm{N}}$. Now assume that $B \neq O_{n}$. Then, as $B$ is symmetric and $\ell_{1}=\mathrm{N}, \operatorname{rank}(B) \geq 2$. We now show that $\operatorname{epr}(B)$ is of one

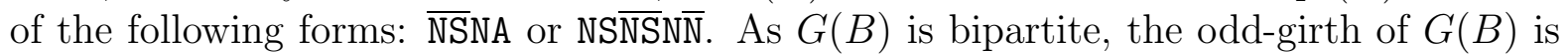
equal to $\infty$. By Theorem [3.4, for all odd $j$ in the interval $[1, n] \cap[1, \infty)=[1, n], \ell_{j}=\mathrm{N}$. Thus, $\operatorname{rank}(B)$ is even (and nonzero). Let $k=\operatorname{rank}(B)-1$. Observe that $k$ is odd and $1 \leq k \leq n-1$ and $1 \leq k<\operatorname{rank}(B)$. By Lemma 3.5, $\ell_{1} \ell_{2} \cdots \ell_{k}$ is of the form $\mathrm{N} \overline{\mathrm{SN}}$, which is of the form $\overline{\mathrm{NSN}}$. As the rank of $B$ is principal, and because $k+1=\operatorname{rank}(B)$, $\ell_{k+1} \in\{\mathrm{A}, \mathrm{S}\}$ and $\ell_{q}=\mathrm{N}$ for all $q \geq k+2$. Observe that $\ell_{k+1}$ is the only term of $\operatorname{epr}(B)$ that remains unknown. If $k=n-1$, then $\operatorname{epr}(B)=\ell_{1} \ell_{2} \cdots \ell_{k+1}$ and, therefore, $\ell_{k+1}=\mathrm{A}$ (because the epr-sequence of no matrix ends with $\mathrm{S}$ ), implying that $\operatorname{epr}(B)$ is of the form NSNA. Now assume that $k \leq n-2$. Then, as NAN is prohibited, $\ell_{k+1}=\mathrm{S}$ and, therefore, $\operatorname{epr}(B)$ is of the form $\overline{\mathrm{NSN}} \mathrm{N} N \overline{\mathrm{N}}$, which is of the form NS$\overline{\mathrm{NS}} \mathrm{N} \overline{\mathrm{N}}$. 
For the other direction, suppose that $\operatorname{epr}(B)$ is of one of the following forms: $N N \bar{N}$ or

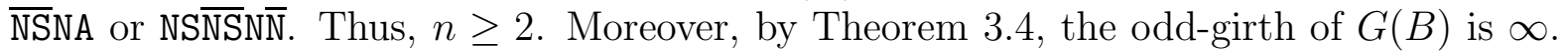
Then, as $G(B)$ is not an isolated vertex (because $n \geq 2), G(B)$ is bipartite.

We now establish statement (2). Suppose that $G(B)=C_{2 k+1}$ for some integer $k \geq 1$. Thus, $n=2 k+1$ (which is an odd integer) and $n \geq 3$. Observe that the odd-girth of $G(B)$ is $n$. Then, by Theorem 3.4. $\ell_{n} \neq \mathrm{N}$. Then, as the epr-sequence of no matrix ends with $\mathrm{S}, \ell_{n}=\mathrm{A}$. By Theorem [3.4, for all odd integers $j$ in the interval $[1, n] \cap[1, n)=[1, n)$, $\ell_{j}=\mathrm{N}$. Since $n-2$ is odd, $\ell_{n-2}=\mathrm{N}$. Then, as $\ell_{n}=\mathrm{A}$, the NN Theorem implies that $\ell_{n-1} \neq \mathrm{N}$. It follows from the fact that NSA is prohibited that $\ell_{n-1}=\mathrm{A}$. It remains to show that $\ell_{i}=\mathrm{S}$ for all even integers with $2 \leq i \leq n-3$; that follows by applying Lemma 3.5 with $k=n-2$.

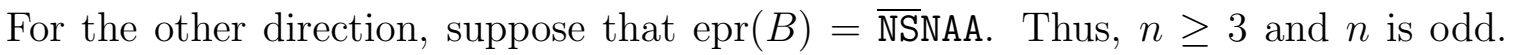
Hence, it suffices to show that $G(B)=C_{n}$. Let $g$ be the odd-girth of $G(B)$. By Theorem 3.4. $g=n$. Thus, $C_{n}$ is a subgraph of $G(B)$. Then, as $G(B)$ is of order $n$, and because $n$ is odd, $G(B)$ does not have any other edges besides those that are part of its cycle of length $n$ (otherwise, $G(B)$ would have an odd cycle that is shorter than $C_{n}=C_{g}$ ). Hence, $G(B)=C_{n}$, as desired.

\section{Symmetric 0-1 matrices that end with NAA}

This section is devoted to symmetric $0-1$ matrices that end with NAA (i.e., to the matrices in the first of the two families associated with Conjecture 1.2).

The particular relevance of the results of the previous section is elucidated by the proof of the next theorem.

Theorem 4.1. Let $n \geq 5$ and $A \in\{0,1\}^{n \times n}$ be symmetric. Suppose that $A$ ends with NAA. Then $A$ ends with either NSNAA or SSNAA. Moreover, if $A$ ends with NSNAA, then $n$ is odd and $A$ is permutationally similar to $A\left(C_{n}\right)$. Consequently, if $n$ is even, then $A$ ends with SSNAA. Furthermore, if $A$ is not permutationally similar to $A\left(C_{n}\right)$, then $A$ ends with SSNAA.

Proof. Let $\operatorname{epr}(A)=\ell_{1} \ell_{2} \cdots \ell_{n}$. Thus, $\ell_{n-2} \ell_{n-1} \ell_{n}=$ NAA. By the NN Theorem, $\ell_{n-3} \neq N$. We claim that $\ell_{n-3}=\mathrm{S}$. Suppose on the contrary that $\ell_{n-3}=$ A. By Theorem 2.11, $\operatorname{epr}(A)=\overline{\text { AAANAA}}$. Thus, $A$ is a $0-1$ matrix that starts with AA, implying that $A=I_{n}$ and, therefore, that $\operatorname{epr}(A)=\mathrm{AAAAA} \overline{\mathrm{A}}$, which is a contradiction. It follows that our claim is true; that is, $\ell_{n-3}=\mathrm{S}$. By Theorem 2.10, $\ell_{n-4} \neq \mathrm{A}$. Thus, $A$ ends with either NSNAA Or SSNAA.

Suppose that $A$ ends with NSNAA. Then, as $A$ is a nonsingular $0-1$ matrix, $A$ does not start with AN (otherwise, $A=J_{n}$, which is singular). By the Inverse Theorem, $\operatorname{epr}\left(A^{-1}\right)$ starts with ANSN and ends with A. It follows from Theorem 3.3 that either epr $\left(A^{-1}\right)=$ ANSN $\overline{S N} A$ or $\operatorname{epr}\left(A^{-1}\right)=$ ANSNAA. If it was the case that $\operatorname{epr}\left(A^{-1}\right)=$ ANSNAA, then the Inverse Theorem would imply that $A$ starts with AN, which would be a contradiction. Thus, epr $\left(A^{-1}\right)=$ ANSNSNA , implying that $n$ is odd, as desired. By the Inverse Theorem, 


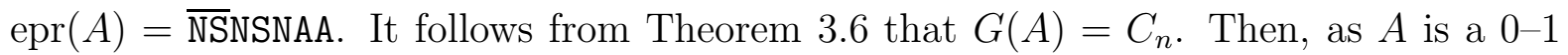
matrix, $A$ is permutationally similar to $A\left(C_{n}\right)$, as desired.

The last two statements of the theorem follow immediately from what was established above.

Observe that Theorem 4.1 implies that the epr-sequences of symmetric $0-1$ matrices of order at least 5 that end with NAA may be partitioned into two classes: those that end with NSNAA and those that end with SSNAA. For any positive integer $k \geq 2, A\left(C_{2 k+1}\right)$ is an example of a symmetric 0-1 matrix that ends with NSNAA (see Theorem 3.6). An example of a symmetric 0-1 matrix that ends with SSNAA, which we borrowed from [2], is the $9 \times 9$ matrix $A\left(C_{3}\right) \otimes A\left(C_{3}\right)$, whose epr-sequence is NSSSSSNAA.

We are ready to establish the main result of this section, which asserts that Conjecture 1.2 holds if the matrix $A$ does not have both a zero and a nonzero principal minor of order $n-4$ (if $n \geq 5$ ).

Theorem 4.2. Let $n \geq 5$ be an integer, let $A \in\{0,1\}^{n \times n}$ be symmetric and nonsingular, and let $\operatorname{epr}(A)=\ell_{1} \ell_{2} \cdots \ell_{n}$. Suppose that $\ell_{n-4} \neq \mathrm{S}$. Then the following statements are equivalent:

(i) $A$ ends with NAA.

(ii) $A^{-1}$ is equimodular with constant diagonal.

Proof. By Remark 1.3, it suffices to show that (i) implies (ii). Suppose that $A$ ends with NAA. By Remark 1.4, it suffices to show that all of the principal minors of order $n-1$ of $A$ are equal. By Theorem 4.1, $A$ ends with NSNAA and $A$ is permutationally similar to $A\left(C_{n}\right)$. Then, as any two induced subgraphs of $C_{n}$ of order $n-1$ are isomorphic, and because $A$ is a $0-1$ matrix, any two principal submatrices of $A$ of order $n-1$ are permutationally similar, implying that any two principal minors of order $n-1$ of $A$ are equal.

With Theorem 4.2 uncovered, Conjecture 1.2 remains unresolved only in the case where the matrix $A$ has the property of having both a zero and a nonzero principal minor of order $n-4$.

We shall now focus on the determinant and order- $(n-1)$ principal minors of symmetric matrices in $\{0,1\}^{n \times n}$ that end with NAA, particularly on their parity; this is for two reasons. In [2, p. 55], the following question was formulated: If $A \in\{0,1\}^{n \times n}$ is a nonsingular symmetric matrix such that $A^{-1} \in\{-\alpha, \alpha\}^{n \times n}$ (i.e., it is equimodular) with constant diagonal, then what are the values that $\alpha$ may attain? By Theorem 2.1, $\alpha$ is either equal to, or the negative of, the ratio of an order- $(n-1)$ principal minor of $A$ to $\operatorname{det}(A)$; this gives us the first reason. To see the second reason, recall that Remark 1.4 implies that, to establish Conjecture 1.2 (if it holds), it suffices to establish the following statement: If $A$ is a symmetric matrix in $\{0,1\}^{n \times n}$ that ends with NAA, then all of the order- $(n-1)$ principal minors of $A$ are equal.

To study the parity of the principal minors of symmetric $0-1$ matrices that end with NAA, we draw upon the author's work in [8]. In Section 1, we defined the epr-sequence 
of a symmetric matrix over $\mathbb{R}$. We now extend that definition to any field $\mathbb{F}$, with the sole purpose of studying a (real) 0-1 matrix by interpreting it as a matrix over $\mathbb{Z}_{2}$. If $A \in\{0,1\}^{n \times n}\left(\subseteq \mathbb{R}^{n \times n}\right)$ is symmetric, then we shall denote by $\operatorname{epr}_{2}(A)$ its epr-sequence as a matrix over $\mathbb{Z}_{2}$. For example, if

$$
A=\left(\begin{array}{lll}
0 & 1 & 1 \\
1 & 0 & 1 \\
1 & 1 & 0
\end{array}\right) \in\{0,1\}^{3 \times 3}\left(\subseteq \mathbb{R}^{3 \times 3}\right),
$$

then $\operatorname{epr}(A)=$ NAA and $\operatorname{epr}_{2}(A)=$ NAN. Observe that, if $A$ is an arbitrary symmetric matrix in $\{0,1\}^{n \times n}\left(\subseteq \mathbb{R}^{n \times n}\right)$ and the $k$ th term of $\operatorname{epr}_{2}(A)$ is $\mathrm{A}$ (respectively, $\mathrm{N}$ ), then all of the principal minors of $A$ of order $k$ are odd (respectively, even); and if the $k$ th term is $\mathrm{S}$, then $A$ has both an odd and an even principal minor of order $k$. [8, Theorems 3.2, $3.8,3.11$ ] provide a complete characterization of the epr-sequences of symmetric matrices over $\mathbb{Z}_{2}$; we draw upon these theorems to establish our next result.

Proposition 4.3. Let $A \in\{0,1\}^{n \times n}$ be symmetric and $\operatorname{epr}(A)=\ell_{1} \ell_{2} \cdots \ell_{n}$. Suppose that $\ell_{n-2} \ell_{n-1} \ell_{n}=\mathrm{NAA}$. Then $\operatorname{epr}_{2}(A)$ is of one of the following forms:

\section{$\overline{\mathrm{NSN}} \mathrm{N}, \quad \overline{\mathrm{NSNNNN}}, \quad$ A $\overline{\mathrm{SNNNN}} \overline{\mathrm{N}}, \quad$ or $\quad \mathrm{S} \overline{\mathrm{SNNNN}} \overline{\mathrm{N}}$.}

Proof. Let $\operatorname{epr}_{2}(A)=\ell_{1}^{\prime} \ell_{2}^{\prime} \cdots \ell_{n}^{\prime}$. Observe that $\ell_{1}^{\prime}=\ell_{1}$. Moreover, for all $j \in[n]$, if $\ell_{j}=\mathrm{N}$, then $\ell_{j}^{\prime}=\mathrm{N}$. Thus, $\ell_{n-2}^{\prime}=\mathrm{N}$. We divide the proof into three cases, based on $\ell_{1}^{\prime}$.

Case 1: $\ell_{1}^{\prime}=\mathrm{N}$.

As $\ell_{n-2}^{\prime}=\mathrm{N}$, it follows from [8, Theorem 3.2] that $\operatorname{epr}_{2}(A)$ is of one of the following

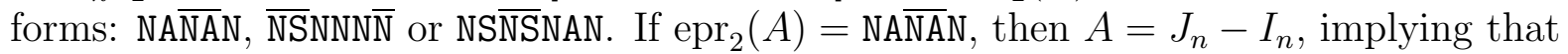
$\operatorname{epr}(A)=\operatorname{NAA} \bar{A}$ (which is not hard to verify) and, therefore, that $n=3$, which implies

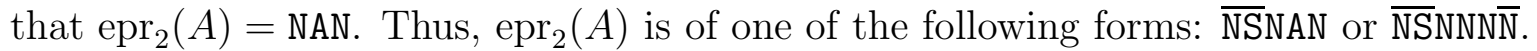

Case 2: $\ell_{1}^{\prime} \neq \mathrm{N}$.

As $\ell_{n-2}^{\prime}=\mathrm{N}$, it follows from [8, Theorem 3.8 and Theorem 3.11] that $\operatorname{epr}_{2}(A)$ is of one of the following forms: A $\bar{S} N N N \bar{N}$ or $S \bar{S} N N N \bar{N}$.

Each of the next two results is a corollary to the result preceding it (they follow readily).

Corollary 4.4. Let $A \in\{0,1\}^{n \times n}$ be symmetric and $\operatorname{epr}(A)=\ell_{1} \ell_{2} \cdots \ell_{n}$. Suppose that $\operatorname{epr}(A)$ ends with NAA. Then $\operatorname{epr}_{2}(A)$ ends with either NAN or NNN. Moreover, if $n$ is even, then $\operatorname{epr}_{2}(A)$ ends with NNN. Moreover, if $\ell_{1} \neq \mathrm{N}$, then $\operatorname{epr}_{2}(A)$ ends with NNN.

Corollary 4.5. Let $A \in\{0,1\}^{n \times n}$ be symmetric and $\operatorname{epr}(A)=\ell_{1} \ell_{2} \cdots \ell_{n}$. Suppose that $A$ ends with NAA. Then the following statements hold:

(i) $\operatorname{det}(A)$ is even.

(ii) If $n$ is even, then all of the principal minors of $A$ of order $n-1$ are even.

(iii) If $\ell_{1} \neq \mathrm{N}$, then all of the principal minors of $A$ of order $n-1$ are even. 
As noted in [2, p. 53], empirical evidence suggests that nonsingular symmetric matrices in $\{0,1\}^{n \times n}$ whose inverses are equimodular with constant diagonal (which, by Remark 1.3, are matrices that end with NAA) are rare. The fact that the product of any two order- $(n-1)$ principal minors of any such matrix is a perfect square (which is a fairly restrictive condition) is supportive of such a hypothesis:

Proposition 4.6. Let $A \in\{0,1\}^{n \times n}$ be symmetric. Suppose that $A$ ends with NAA. Then the product of any two principal minors of $A$ of order $n-1$ is a perfect square.

Proof. Let $i, j \in[n]$. Let $d_{i}=\operatorname{det}(A(\{i\})), d_{j}=\operatorname{det}(A(\{j\}))$ and $d_{i j}=\operatorname{det}(A(\{i\},\{j\}))$. We now show that $d_{i} d_{j}=d_{i j}{ }^{2}$. As the desired conclusion follows immediately if $i=j$, we assume that $i \neq j$. Observe that $A$ is nonsingular (because it ends with A). Let $A^{-1}=$ $\left[\alpha_{i j}\right]$. By the Inverse Theorem, $A^{-1}$ starts with AN. Thus, $0=A^{-1}[\{i, j\}]=\alpha_{i i} \alpha_{j j}-\alpha_{i j}^{2}$, implying that $\alpha_{i i} \alpha_{j j}=\alpha_{i j}^{2}$. As $A$ is symmetric, $d_{i j}=\operatorname{det}(A(\{j\},\{i\}))$. It follows from Theorem 2.1 that $d_{i} d_{j}=d_{i j}^{2}$.

Observe that Proposition 4.6 holds regardless of whether or not $A$ is a $0-1$ matrix.

\section{$5 \quad 0$-1 matrices whose inverses are equimodular}

This section focuses on nonsingular 0-1 matrices whose inverses are equimodular, due to their relation to the second of the two families of matrices associated with Conjecture 1.2 (namely, the family of nonsingular symmetric 0-1 matrices whose inverses are equimodular with constant diagonal). In Sections 3 and 4, all of the matrices involved were symmetric, as we are motivated by Conjecture 1.2. However, in this section, in Subsection 5.1, we shall start by establishing results that apply not only to symmetric matrices but also to non-symmetric matrices, and then, in Subsection 5.2, we shall revert our attention to symmetric matrices. The present section will conclude with summative remarks about the section, in Subsection 5.3, by looking at its results through the lens of Conjecture 1.2 .

\subsection{Not necessarily symmetric matrices}

The next fact, which generalizes [2, Proposition 5], will help us cast some light upon the structure of nonsingular 0-1 matrices whose inverses are equimodular, and its proof, which we include merely for completeness, is the same as that of [2, Proposition 5].

Proposition 5.1. Let $n \geq 2$ be an integer and $A \in \mathbb{R}^{n \times n}$ be nonsingular. Suppose that each nonzero entry of $A$ is an odd integer. Suppose that $A^{-1}=q N$, for some rational number $q$ and some matrix $N$ all of whose entries are odd. Then each row, and each column, of $A$ has an even number of nonzero entries.

Proof. Let $A=\left[\vec{a}_{1}, \vec{a}_{2}, \ldots, \vec{a}_{n}\right]$ and $N^{T}=\left[\vec{w}_{1}, \vec{w}_{2}, \ldots, \vec{w}_{n}\right]$. Let $j \in[n]$ and $m$ be the number of nonzero entries of $\vec{a}_{j}$. Let $i \in[n] \backslash\{j\}$. Observe that $\vec{w}_{i}^{T} \vec{a}_{j}$ is the $(i, j)$-entry of $N A$. Then, as $N A$ is a diagonal matrix, and because $i \neq j, \vec{w}_{i}^{T} \vec{a}_{j}=0$, implying that 
$\vec{w}_{i}^{T} \vec{a}_{j}$ is even. As $\vec{w}_{i}^{T} \vec{a}_{j}$ is the sum of $m$ odd numbers, the parity of $\vec{w}_{i}^{T} \vec{a}_{j}$ is the same as that of $m$. It follows that $m$ is even. Thus, each column of $A$ has an even number of nonzero entries.

Applying what was established in the previous paragraph to $A^{T}$ shows that each column of $A^{T}$ has an even number of nonzero entries, implying that each row of $A$ has an even number of nonzero entries.

Each row and column of a 0-1 matrix (of order at least 2) whose inverse is equimodular has an even number of nonzero entries:

Corollary 5.2. Let $n \geq 2$ be an integer and $A \in\{0,1\}^{n \times n}$ be nonsingular. Suppose that $A^{-1}$ is equimodular. Then each row, and each column, of $A$ has an even number of nonzero entries.

Proof. It is clear that $A^{-1}$ being equimodular implies that $A^{-1}=\alpha N$, for some number $\alpha$ and some matrix $N \in\{-1,1\}^{n \times n}$. Observe that each entry of $N$ is odd. By Theorem 2.1, the fact that each entry of $A$ is rational implies that each entry of $A^{-1}$ is as well, implying that $\alpha$ is rational. It follows from Proposition 5.1 that each row, and each column, of $A$ has an even number of nonzero entries.

The determinants of nonsingular 0-1 matrices whose inverses are equimodular are all even:

Theorem 5.3. Let $n \geq 2$ be an integer and $A \in\{0,1\}^{n \times n}$ be nonsingular. Suppose that $A^{-1}$ is equimodular, with $A^{-1} \in\{-\alpha, \alpha\}^{n \times n}$, for some $\alpha$. Then the following statements hold:

1. $\alpha=\frac{1}{2 m}$, for some integer $m$.

2. Each minor of $A$ of order $n-1$ divides $\operatorname{det}(A)$.

3. $\operatorname{det}(A)$ is even.

Proof. As $A^{-1} \in\{-\alpha, \alpha\}^{n \times n}$, there exists a matrix $N \in\{-1,1\}^{n \times n}$ such that $A^{-1}=\alpha N$. As $A^{-1}$ is nonsingular, $\alpha \neq 0$. Thus, $A N=\frac{1}{\alpha} I_{n}$. By Corollary [5.2, each row of $A$ has an even number of nonzero entries. Then, as $A \in\{0,1\}^{n \times n}$ and $N \in\{-1,1\}^{n \times n}$, each entry of $A N$ is the sum of an even number of odd numbers; as such a sum is even, each entry of $A N$ is even. Then, as $A N=\frac{1}{\alpha} I_{n}, \frac{1}{\alpha}$ is an even integer. Hence, $\alpha=\frac{1}{2 m}$, for some integer $m$, establishing statement (11).

Let $d=\operatorname{det}(A)$ and $t$ be a minor of $A$ of order $n-1$. As $A^{-1} \in\{-\alpha, \alpha\}^{n \times n}$, Theorem 2.1 implies that $\left|\frac{t}{d}\right|=|\alpha|$. Thus, $\left|\frac{d}{t}\right|=|2 m|$. Then, as $d$ and $t$ are integers (because $A$ is a 0-1 matrix), $t$ divides $d$. Hence, statement (2) follows. As $|d|=|2 m t|$, and because $d$ and $t$ are integers, statement (3) holds.

We note that Theorem 5.3 asserts that not only all principal minors of $A$ of order $n-1$ divide $\operatorname{det}(A)$, but also all non-principal minors of $A$ of order $n-1 \operatorname{divide} \operatorname{det}(A)$.

Due to the seeming irrelevance of the next fact, it is worthwhile to note that its inclusion will be justified at the end of this section (in Remark 5.14). 
Proposition 5.4. Let $n \geq 2$ be an integer and $A \in\{0,1\}^{n \times n}$ be nonsingular. Suppose that $A^{-1}$ is equimodular, with $A^{-1} \in\{-\alpha, \alpha\}^{n \times n}$, for some $\alpha$. Suppose that $|\operatorname{det}(A)|=$ $2^{k}$, for some integer $k>0$. Then there exists an integer $t$ with $0 \leq t<k$ such that the modulus of each minor of $A$ of order $n-1$ is $2^{t}$. Moreover, $|\alpha|=\frac{1}{2^{k-t}}$. Moreover, if $A$ has an odd minor of order $n-1$, then $t=0$ and $|\alpha|=\left|\frac{1}{\operatorname{det}(A)}\right|$.

Proof. As $A^{-1}$ is equimodular, Theorem 2.1 implies that the moduli of any two minors of $A$ of order $n-1$ are equal. By Theorem 5.3, each minor of $A$ of order $n-1$ divides $\operatorname{det}(A)$. Thus, there exists an integer $t$ with $0 \leq t \leq k$ such that the modulus of each minor of $A$ of order $n-1$ is $2^{t}$. By Theorem [2.1, $|\alpha|=\frac{2^{t}}{2^{k}}=\frac{1}{2^{k-t}}$. By Theorem [5.3, $|\alpha|$ is the reciprocal of an even integer. Thus, $t \neq k$, implying that $0 \leq t<k$, as desired. The last statement follows readily from the statements preceding it.

The next result asserts that the inverse of a Schur complement in a nonsingular 0-1 matrix whose inverse is equimodular is itself equimodular, and it follows immediately from the Schur Complement Theorem.

Proposition 5.5. Let $n \geq 2$ be an integer and $A \in\{0,1\}^{n \times n}$ be nonsingular, and let $\mu \subset$ $[n]$. Suppose that $A[\mu]$ is nonsingular, and let $C=A / A[\mu]$. If $A^{-1}$ is equimodular, then $C^{-1}$ is equimodular. Furthermore, if $A^{-1} \in\{-\alpha, \alpha\}^{n \times n}$, then $C^{-1} \in\{-\alpha, \alpha\}^{(n-|\mu|) \times(n-|\mu|)}$.

We note that the statement of Proposition 5.5 would continue to hold if $A$ was replaced with an arbitrary nonsingular matrix in $\mathbb{R}^{n \times n}$.

Lemma 5.6. Let $n \geq 3$ be an integer, and let $A \in\{0,1\}^{n \times n}$ be nonsingular and of the form

$$
A=\left(\begin{array}{c|c}
1 & \vec{y}^{T} \\
\hline \vec{x} & Z
\end{array}\right),
$$

for some $\vec{x}, \vec{y} \in \mathbb{R}^{n-1}$ and $Z \in \mathbb{R}^{(n-1) \times(n-1)}$. Suppose that $A^{-1}$ is equimodular. Then each row, and each column, of $Z-\vec{x} \vec{y}^{T}$ has an even number of nonzero entries.

Proof. Let $C=Z-\vec{x} \vec{y}^{T}$ and $\mu=\{1\}$. Observe that $Z=A\left[\mu^{c}\right]$ (where $\mu^{c}=[n] \backslash \mu$ ), that $\vec{x}=A\left[\mu^{c}, \mu\right]$, that $\vec{y}^{T}=A\left[\mu, \mu^{c}\right]$, that $A[\mu]$ is the $1 \times 1$ matrix [1], which is nonsingular, and that $(A[\mu])^{-1}=[1]$. It follows, then, that $C=A / A[\mu]$. As $A^{-1}$ is equimodular, Proposition 5.5 implies that $C^{-1}$ is equimodular as well, implying that $C^{-1}=\alpha N$, for some number $\alpha$ and some $N \in\{-1,1\}^{(n-1) \times(n-1)}$. As each entry of $C$ is rational, Theorem 2.1 implies that each entry of $C^{-1}$ is also rational and, therefore, that $\alpha$ is rational. As $Z$ and $\vec{x} \vec{y}^{T}$ are both $0-1$ matrices, $C \in\{-1,0,1\}^{(n-1) \times(n-1)}$. Then, as $n-1 \geq 2$, Proposition 5.1 implies that each row, and each column, of $C$ has an even number of nonzero entries.

Our next result casts light upon the structure of nonsingular $0-1$ matrices whose inverses are equimodular.

Theorem 5.7. Let $n \geq 3$ be an integer and $A \in\{0,1\}^{n \times n}$ be nonsingular, and let $A=\left[\vec{a}_{1}, \vec{a}_{2}, \ldots, \vec{a}_{n}\right]$ and $A^{T}=\left[\vec{r}_{1}, \vec{r}_{2}, \ldots, \vec{r}_{n}\right]$. Suppose that $A^{-1}$ is equimodular. Then each of $\vec{a}_{i}-\vec{a}_{j}$ and $\vec{r}_{i}-\vec{r}_{j}$ has an even number of nonzero entries, for all $i, j \in[n]$. 
Proof. Let $A=\left[a_{i j}\right]$. We start by showing that $\vec{a}_{i}-\vec{a}_{j}$ has an even number of nonzero entries, for all $i, j \in[n]$. Let $i, j \in[n]$. As the desired conclusion follows immediately if $i=j$, assume that $i \neq j$. We consider two cases, based on $\vec{a}_{i}^{T} \vec{a}_{j}$.

Case 1: $\vec{a}_{i}^{T} \vec{a}_{j}=0$.

Let $m$ and $\ell$ be the number of nonzero entries of $\vec{a}_{i}$ and $\vec{a}_{j}$, respectively. As $\vec{a}_{i}^{T} \vec{a}_{j}=0$, and because $\vec{a}_{i}$ and $\vec{a}_{j}$ are $0-1$ vectors, the number of nonzero entries of $\vec{a}_{i}-\vec{a}_{j}$ is $m+\ell$. By Corollary 5.2, $m$ and $\ell$ are both even, implying that $m+\ell$ is even, as desired.

Case 2: $\vec{a}_{i}^{T} \vec{a}_{j} \neq 0$.

As $\vec{a}_{i}$ and $\vec{a}_{j}$ are $0-1$ vectors, there exists $k \in[n]$ such that $a_{k i}=a_{k j}=1$ (otherwise, $\left.\vec{a}_{i}^{T} \vec{a}_{j}=0\right)$. Let $P \in \mathbb{R}^{n \times n}$ be a permutation matrix such that the first row of $P A$ is the $k$ th row of $A$. Let $Q \in \mathbb{R}^{n \times n}$ be a permutation matrix such that the first and second columns of $P A Q$ are, respectively, the $i$ th and $j$ th columns of $P A$. Let $B=P A Q$, $B=\left[\vec{b}_{1}, \vec{b}_{2}, \ldots, \vec{b}_{n}\right]$ and $B=\left[b_{i j}\right]$. Observe that the number of nonzero entries of $\vec{a}_{i}-\vec{a}_{j}$ is equal to the number of nonzero entries of $\vec{b}_{1}-\vec{b}_{2}$. Thus, it suffices to show that $\vec{b}_{1}-\vec{b}_{2}$ has an even number of nonzero entries. Observe that $b_{11}=1$ (because $a_{k i}=1$ ) and $b_{12}=1$ (because $a_{k j}=1$ ). Thus, $B$ is of the form

$$
B=\left(\begin{array}{c|c}
1 & \vec{y}^{T} \\
\hline \vec{x} & Z
\end{array}\right),
$$

for some $\vec{x}, \vec{y} \in \mathbb{R}^{n-1}$ and $Z \in \mathbb{R}^{(n-1) \times(n-1)}$. Let $\vec{y}=\left[y_{1}, \ldots, y_{n-1}\right]^{T}$ and $Z=\left[\vec{z}_{1}, \ldots, \vec{z}_{n-1}\right]$. Then, as $y_{1}=b_{12}=1$,

$$
\vec{b}_{1}-\vec{b}_{2}=\left(\frac{1-y_{1}}{\vec{x}-\vec{z}_{1}}\right)=-\left(\frac{0}{\vec{z}_{1}-\vec{x}}\right)
$$

Thus, it suffices to show that $\vec{z}_{1}-\vec{x}$ has an even number of nonzero entries. Observe that $B^{-1}=Q^{T} A^{-1} P^{T}$. It follows, then, that $B^{-1}$ is equimodular (because $A^{-1}$ is equimodular). Then, as $B \in\{0,1\}^{n \times n}$, and because $n \geq 3$, Lemma 5.6 implies that $\vec{z}_{1}-y_{1} \vec{x}$, the first column of $Z-\vec{x} \vec{y}^{T}$, has an even number of nonzero entries; as $y_{1}=1, \vec{z}_{1}-\vec{x}$ has an even number of nonzero entries, as desired.

Let $M=A^{T}$. Observe that $M \in\{0,1\}^{n \times n}$, that $M$ is nonsingular, that $M=$ $\left[\vec{r}_{1}, \vec{r}_{2}, \ldots, \vec{r}_{n}\right]$, and that $M^{-1}$ is equimodular $\left(M^{-1}=\left(A^{-1}\right)^{T}\right)$. It follows from what was established in the previous paragraph that, for all $i, j \in[n], \vec{r}_{i}-\vec{r}_{j}$ has an even number of nonzero entries.

\subsection{Symmetric matrices}

We shall now revert our attention to symmetric matrices, albeit with our focus still on $0-1$ matrices whose inverses are equimodular.

Lemma 5.8. Let $B \in \mathbb{R}^{n \times n}$ be symmetric. Suppose that each row of $B$ has an even number of nonzero entries. Then $B$ has an even number of nonzero diagonal entries. 
Proof. Let $m$ be the number of nonzero diagonal entries of $B$. Let $M$ be the matrix that results from replacing each nonzero diagonal entry of $B$ with 0 . Then, as each row of $B$ has an even number of nonzero entries, $M$ has exactly $m$ rows with an odd number of nonzero entries. It follows, then, that $G(M)$ has exactly $m$ vertices of odd degree. By the (well-known) degree-sum formula, $m$ is even.

A nonsingular symmetric 0-1 matrix (of order at least two) whose inverse is equimodular has an even number of nonzero diagonal entries:

Theorem 5.9. Let $n \geq 2$ be an integer, and let $A \in\{0,1\}^{n \times n}$ be symmetric and nonsingular. Suppose that $A^{-1}$ is equimodular. Then $A$ has an even number of nonzero diagonal entries.

Proof. By Corollary 5.2, each row of $A$ has an even number of nonzero entries. The desired conclusion follows from Lemma 5.8.

Lifting the assumption that $A$ is symmetric in Theorem 5.9 renders the statement of the proposition false:

Example 5.10. Observe that the inverse of the non-symmetric 0-1 matrix

$$
A=\left(\begin{array}{lll}
1 & 1 & 0 \\
0 & 1 & 1 \\
1 & 0 & 1
\end{array}\right) \quad \text { is } \quad A^{-1}=\frac{1}{2}\left(\begin{array}{ccc}
1 & -1 & 1 \\
1 & 1 & -1 \\
-1 & 1 & 1
\end{array}\right)
$$

which is equimodular. As $A$ has an odd number of nonzero diagonal entries, we conclude that a non-symmetric 0-1 matrix whose inverse is equimodular need not have an even number of nonzero diagonal entries.

The inclusion of the following observation will be justified shortly.

Observation 5.11. Let $A \in\{0,1\}^{n \times n}$ be symmetric, $A=\left[a_{i j}\right]$ and $A=\left[\vec{a}_{1}, \vec{a}_{2}, \ldots, \vec{a}_{n}\right]$. Let $k \in[n], B=A-\vec{a}_{k} \vec{a}_{k}^{T}$ and $B=\left[\vec{b}_{1}, \vec{b}_{2}, \ldots, \vec{b}_{n}\right]$. Then, for all $i \in[n]$,

$$
\vec{b}_{i}= \begin{cases}\vec{a}_{i}-\vec{a}_{k}, & \text { if } a_{i k}=1 \\ \vec{a}_{i}, & \text { if } a_{i k}=0\end{cases}
$$

The purpose of making the observation above (Observation 5.11) is to note that knowledge of the structure of $A$ may be revealed through the structure of $A-\vec{a}_{k} \vec{a}_{k}^{T}$, as the matrix $A-\vec{a}_{k} \vec{a}_{k}^{T}$ is obtained from $A$ by simply subtracting $\vec{a}_{k}$ from certain column vectors of $A$; this observation is rendered useful, for us, by the following theorem, which should be viewed through the lens of Observation 5.11 .

Theorem 5.12. Let $n \geq 3$ be an integer, let $A \in\{0,1\}^{n \times n}$ be symmetric and nonsingular, and let $A=\left[\vec{a}_{1}, \vec{a}_{2}, \ldots, \vec{a}_{n}\right]$. Suppose that $A^{-1}$ is equimodular. Then $A-\vec{a}_{k} \vec{a}_{k}^{T}$ has an even number of nonzero diagonal entries, for all $k \in[n]$. 
Proof. Let $k \in[n], B=A-\vec{a}_{k} \vec{a}_{k}^{T}$ and $B=\left[\vec{b}_{1}, \vec{b}_{2}, \ldots, \vec{b}_{n}\right]$. It follows from Observation 5.11 that, for all $i \in[n]$, either $\vec{b}_{i}=\vec{a}_{i}-\vec{a}_{k}$ or $\vec{b}_{i}=\vec{a}_{i}$. Thus, by Corollary 5.2 and Theorem 5.7, each column of $B$ has an even number of nonzero entries. Then, as $B$ is symmetric, each row of $B$ has an even number of nonzero entries. By Lemma 5.8, $B$ has an even number of nonzero diagonal entries.

Unsurprisingly, the assumption that $A$ is symmetric in Theorem 5.12 is important:

Example 5.13. Let $A$ be the $3 \times 3$ matrix in Example [5.10, and let $A=\left[\vec{a}_{1}, \vec{a}_{2}, \vec{a}_{3}\right]$. Observe that $A$ is a non-symmetric $0-1$ matrix whose inverse is equimodular, and that

$$
A-\vec{a}_{1} \vec{a}_{1}^{T}=\left(\begin{array}{ccc}
0 & 1 & -1 \\
0 & 1 & 1 \\
0 & 0 & 0
\end{array}\right) .
$$

Thus, $A-\vec{a}_{1} \vec{a}_{1}^{T}$ has an odd number of nonzero diagonal entries. We conclude that relaxing the hypothesis of Theorem 5.12 by not requiring the matrix $A$ to be symmetric leads to a false statement.

\subsection{Summative remarks}

We shall now provide some summative remarks about this section (Section (5)). It is clear that the main results of this section apply to the matrices in the family of nonsingular symmetric $0-1$ matrices whose inverses are equimodular with constant diagonal. Although the results of this section apply to other matrices besides those in this family, we shall keep this family at the center of our summative remarks below, due to its relation to Conjecture 1.2, which was our primary motivation for investigating (not necessarily symmetric) 0-1 matrices whose inverses are equimodular (with not necessarily constant diagonal) in this section.

Hitherto, what appears on the literature regarding matrices in the family of nonsingular symmetric $0-1$ matrices whose inverses are equimodular with constant diagonal is largely focused on those that have zero diagonal (which may be interpreted as adjacency matrices of graphs without loops). On the other hand, of those that have at least one nonzero diagonal entry (examples of which are the last three matrices in [2, p. 54]), not much is known, apart from what is implied by [2, Proposition 5] (i.e., that each of their rows and columns has an even number of nonzero entries). It is, therefore, worthwhile to note that the results uncovered in this section apply to arbitrary nonsingular symmetric 0-1 matrices whose inverses are equimodular with constant diagonal (meaning that there is no restriction imposed on their diagonals); of particular relevance are Theorems 5.7, 5.9 and 5.12, as they cast light upon the structure of these matrices (Theorem 5.12 should be viewed through the lens of Observation 5.11).

The following remark, whose verification is a trivial computational exercise (albeit with the help of [2, p. 53-54]), will help us elucidate the relevance of Proposition 5.4, by linking it to the family of nonsingular symmetric $0-1$ matrices whose inverses are equimodular with constant diagonal. 
Remark 5.14. Let $3 \leq n \leq 9$ be an integer, and let $A \in\{0,1\}^{n \times n}$ be symmetric and nonsingular. Suppose that $A^{-1}$ is equimodular with constant diagonal. Then $|\operatorname{det}(A)|=$ $2^{k}$, for some integer $k>0$. Moreover, if $A$ is neither permutationally similar to $A\left(C_{3}\right) \otimes$ $A\left(C_{3}\right)$ nor permutationally similar to one of the last two $9 \times 9$ matrices on [2, p. 54], then $A$ has an odd minor of order $n-1$.

Up to permutation similarity, there is a total of fifteen nonsingular symmetric 0-1 matrices of order at least 3 and at most 9 whose inverses are equimodular with constant diagonal [2, p. 53-54] (each of these fifteen matrices appears in 2]). Remark [5.14 asserts that, if $A \in\{0,1\}^{n \times n}$ is one of the aforementioned fifteen matrices but is neither permutationally similar to $A\left(C_{3}\right) \otimes A\left(C_{3}\right)$ nor permutationally similar to one of the last two $9 \times 9$ matrices on [2, p. 54], then $A$ satisfies all of the hypotheses of Proposition 5.4 and, therefore, $|\alpha|=\left|\frac{1}{\operatorname{det}(A)}\right|$, where $A^{-1} \in\{-\alpha, \alpha\}^{n \times n}$.

\section{References}

[1] W. Barrett, S. Butler, M. Catral, S. M. Fallat, H. T. Hall, L. Hogben, P. van den Driessche, M. Young. The principal rank characteristic sequence over various fields. Linear Algebra and its Applications 459 (2014), 222-236.

[2] W. Barrett, S. Butler, H. T. Hall. Symmetric 0-1 matrices with inverses having two distinct values and constant diagonal. Linear Algebra and its Applications 491 (2016), 41-55.

[3] R. A. Brualdi, L. Deaett, D. D. Olesky, P. van den Driessche. The principal rank characteristic sequence of a real symmetric matrix. Linear Algebra and its Applications 436 (2012), 2137-2155.

[4] R. A. Brualdi, H. Schneider. Determinantal identities: Gauss, Schur, Cauchy, Sylvester, Kronecker, Jacobi, Binet, Laplace, Muir, and Cayley. Linear Algebra and its Applications 52/53 (1983), 769-791.

[5] S. Butler, M. Catral, S. M. Fallat, H. T. Hall, L. Hogben, P. van den Driessche, M. Young. The enhanced principal rank characteristic sequence. Linear Algebra and its Applications 498 (2016), 181-200.

[6] S. Butler, M. Catral, H. T. Hall, L. Hogben, X. Martínez-Rivera, B. Shader, P. van den Driessche. The enhanced principal rank characteristic sequence for Hermitian matrices. Electronic Journal of Linear Algebra 32 (2017), 58-75.

[7] X. Martínez-Rivera. Classification of families of pr- and epr-sequences. Linear and Multilinear Algebra 65 (2017), 1581-1599.

[8] X. Martínez-Rivera. The enhanced principal rank characteristic sequence over a field of characteristic 2. Electronic Journal of Linear Algebra 32 (2017), 273-290. 
[9] F. Zhang (editor). The Schur Complement and its Applications. Springer-Verlag, New York, New York, 2005. 